Bull. Egypt. Soc. Physiol. Sci. 37(1),59-68

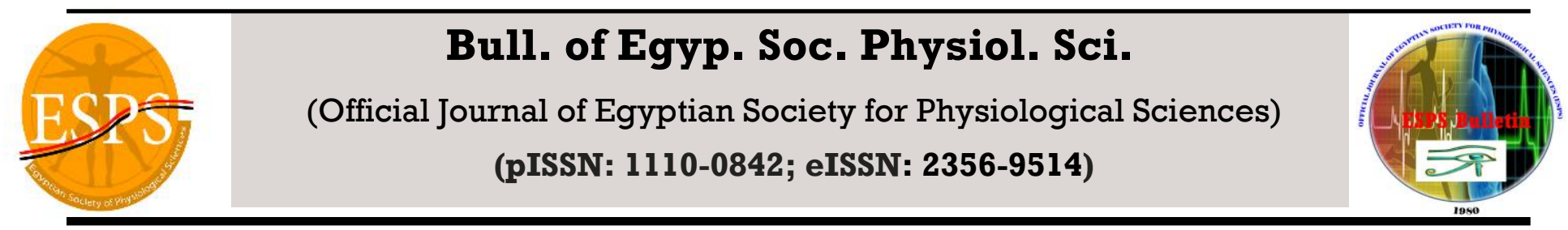

\title{
Value of Serum miRNA 146a as Biomarker for Early Diagnosis of Neonatal Sepsis
}

\author{
Inas A. Ahmed ${ }^{1,2}$ Amal Abou Elfadle ${ }^{1}$ and Ghada Saad Abdelmotaleb ${ }^{3}$ \\ ${ }^{1}$ Department of Medical Biochemistry, ${ }^{2}$ Molecular Biology and Biotechnology Unit, ${ }^{3}$ Department of Pediatrics, \\ Faculty of Medicine, Benha University
}

Received: 28 Dec 2016

Accepted: 31 Jan 2017

Available online: 29 Mar 2017

\section{Keywords}

- Neonatal sepsis

- miRNAs

- Systemic

inflammatory

response syndrome

\begin{abstract}
Neonatal sepsis (NS) is regarded as one of the most common causes of morbidity and mortality among neonates worldwide. However, the magnitude of the problem is higher in low income countries, in spite of recent improvement of neonatal health care units. Accurate and early diagnosis of NS is the corner stone toward better therapeutic approach and safe outcome. Creactive protein is currently used however it has been reported to have a low specificity and to rise in blood of cases with a delay of 24 hours than other biomarkers like inerleukin-16 or procalcitonin. Therefore, identification of new biomarkers with high specificity and sensitivity is extremely needed. The current study aimed to detect changes in miR-146a serum expression levels among NS cases, neonates at high risk to develop sepsis and healthy control neonates and to assess the value of serum miR-146a as early indicator of NS. qPCR was used for detection of miR-146a serum expression among different study groups. Serum miR-146a showed differential expression of statistical significance within study groups, with the highest level in NS group and the lowest in healthy controls. Moreover, serum miR-146a showed a significant positive correlation with the CRP titer in NS group. Receiver operating characteristic (ROC) curve analysis revealed that best cut off value for miR-146a was at 0.539 relative units, with a sensitivity of $91.7 \%$, specificity of $100 \%$ and $95.7 \%$ accuracy. As regard to CRP, the best ROC curve was at $18 \mathrm{mg} / \mathrm{dL}$ with $100 \%$ sensitivity, $86.4 \%$ specificity and $93.5 \%$ accuracy. Combination of miR-146a and CRP increased specificity, sensitivity and accuracy to $100 \%$. Furthermore, in multivariate logistic regression analysis, only CRP and miR-146a were considered as independent risk factor for prediction of sepsis within high risk neonates. In conclusion, serum miR-146a may be a promising predictor for sepsis within the high risk neonates. However, large-scale prospective studies are required to confirm our findings.
\end{abstract}

Corresponding author: Inas Ahmed . Department of Medical Biochemistry, Molecular Biology and Biotechnology Unit, Faculty of Medicine, Benha University, Al Qalyubia, Egypt, PO 13518.; email: inas.ahmed@ fmed.bu.edu.eg 


\section{Introduction}

Neonatal sepsis is defined as systemic inflammatory response syndrome (SIRS) in the presence of suspected or proven infection, (according to the 2002 International Pediatric Sepsis Consensus Conference) (1). Based on the age at onset, NS is further classified into earlyonset neonatal sepsis (EOS) $<7$ days in term infants, and late onset sepsis (LOS) afterwards. In EOS cases, the causative pathogens are transmitted from maternal side trans-placentally or during delivery, while LOS cases may be due to pathogens acquired from environment as well (2).

Despite the advances in neonatal health care, a recent study reported that the total mortality rate for the proven neonatal sepsis in Egypt, was 51\%. Importantly, the NS survivors are at high risk for neurological and developmental morbidity (3).

Early diagnosis of NS and efficient therapeutic approach may help to improve the prognosis and outcome of NS cases. However early identification of NS is a great challenge due to the nonspecific clinical presentations. So far, blood culture is the main laboratory confirmatory test, however, it is a time-consuming procedure and its results may be affected by antibiotic administration. Moreover, acute phase reactants as $\mathrm{C}$-reactive protein (CRP) and cytokines lack the ability to specifically differentiate between NS and SIRS or other inflammatory diseases. In addition, CRP plasma level increases with a lag of 24 hours after cytokines and procalcitonin $(\mathbf{4 , 5}$ ).

On the other hand, initiation of empirical antibiotic treatment once NS is suspected, increases the risk of development of multidrug resistant pathogens
(3). Hence, the urgent need for reliable inflammatory markers for rapid and accurate identification of NS cases(6).

MicroRNAs (miRNAs) are small, non-coding RNAs that regulate gene expression at a posttranscriptional level via mediating mRNA cleavage, translational repression or mRNA destabilization (7).

Circulating miRNAs represent a promising tool for diagnostic purpose in many diseases with several advantages including easy detection, rapid and accurate measurement. Surprisingly, circulating miRNAs are more stable than other RNAs, as they withstand many conditions like $\mathrm{pH}$ changes, repeated freezing/thawing and prolonged storage periods. The nature of circulating miRNAs explains this stability, because they are transferred in the form of lipoprotein complexes or encapsulated within exosomes $(\mathbf{4}, \mathbf{8})$. Moreover, compared to conventional protein-based markers, miRNAs are smaller with a more simple chemical structure (8).

MiR-146a is a member of the miR-146a miRNA family, it plays an important role in regulating inflammatory responses via a negative feedback pathway. MiR-146a is differentially expressed in several inflammatory, autoimmune diseases, viral infections and cancer. Therefore, previous studies suggested its use as a biomarker for diagnostic, prophylactic and therapeutic purposes(9).

The current study aimed to detect changes in gene expression level of serum miR-146a in NS cases, newborn at high risk to develop sepsis and healthy control neonates, to assess its potential use as indicator for early diagnosis of neonatal sepsis. 


\section{Subjects and Methods}

The current study was conducted after approval of the study scheme by the ethical committee of Faculty of Medicine, Benha University. It included 24 neonates with clinical manifestations suggestive of NS and 22 neonates who were at high risk to develop sepsis. Additional 17 apparently healthy neonates were recruited as controls and showed no clinical or laboratory evidence suggestive of sepsis. The subjects were selected from the Neonatal Intensive Care Unit (NICU) of Department of Pediatrics, Benha University Hospital, after obtaining an informed consent from the legal guardians of the neonates. The clinical data suggesting sepsis included temperature instability (hypothermia, hyperthermia), respiratory distress, cardiovascular (bradycardia), neurological (seizures) and gastrointestinal dysfunction symptoms and signs (vomiting, diarrhea, abdominal distention, jaundice). Diagnosis was established according to clinical findings and laboratory results of CRP titer and blood culture. The risk factors for NS included premature rupture of membrane $(\mathrm{PROM})>18$ hours, maternal fever $>38{ }^{\circ} \mathrm{C}$, vaginal bleeding, chorioamnionitis, maternal use of antibiotics, maternal urinary tract infection (UTI). Neonates suffered from severe congenital anomalies, chromosomal abnormalities, intrauterine growth retardation, perinatal asphyxia and infants of diabetic mothers were excluded.

\section{Sampling:}

Peripheral blood samples $(3 \mathrm{ml})$ were withdrawn from the neonates during collection of first blood cultures and divided into 2 parts. The first part (1ml) was collected in EDTA tubes for CBC, performed by automated hematology analyzer Sysmex XS-1000i (Sysmex, Japan). The second part $(2 \mathrm{ml})$ was collected into sterile vacutainers, allowed to clot, then serum was separated by centrifugation at room temperature for 10 minutes at 2,000 xg. The separated sera were used for CRP titer detection using latex agglutination (CRPLatex Cromatest) (10) and the remaining were stored at $-80^{\circ} \mathrm{C}$ for later miRNA isolation.

\section{Isolation of total RNA including miRNA:}

miRNeasy Serum/Plasma Kit (Qiagen, Germany) was used according to the manufacturer's instructions to separate total RNA including miRNA from serum samples.

\section{cDNA synthesis:}

The concentration of RNA was detected in each sample by Nanodrop 2000c spectrophotometer (Thermo Scientific, USA), and $(1 \mu \mathrm{g})$ was used for cDNA synthesis by G-storm thermal cycler (Thermo, England). Reverse transcription was performed using miScript II RT Kit (Qiagen, Germany) according to the manufacturer's instructions. The cDNA samples were kept frozen at $-20^{\circ} \mathrm{C}$ for later $\mathrm{qPCR}$ analysis.

\section{Relative quantitation of miR-146a:}

qPCR was performed by StepOne ${ }^{\mathrm{TM}}$ Real-Time PCR System (Life Technologies, USA). miScript SYBR Green PCR Kit (Qiagen, Germany), was used for miR-146a assays. According to the $2^{-\Delta \Delta \mathrm{Ct}}$ method, quantification of miR-146a was expressed as relative quantitative values after normalization with RNU6 gene(11). Hs-miR-146a$5 \mathrm{p}$ and RNU6-2 miScript Primer Assays were 
supplied by Qiagen, Germany for miR-146a qPCR assays.

\section{Statistical analysis}

The collected data were tabulated and analyzed using SPSS version 20 software (SPSS Inc, Chicago, ILL Company). Categorical data were presented as number and percentages while quantitative data were expressed as mean \pm standard deviation or median, and range. Chi square $\left(\mathrm{X}^{2}\right)$ test, Fisher's exact test, Man Whitney $\mathrm{U}$ test, and Spearman's correlation coefficient were used as tests of significance. ROC curve was used to determine cutoff value of miR-146a with optimum sensitivity and specificity in early diagnosis of sepsis. Logistic regression analysis was used for prediction of sepsis. The accepted level of significance in this work was stated at 0.05 ( $\mathrm{P}<0.05$ was considered significant).

\section{Results}

The current study included 24 neonates who were diagnosed as proved sepsis, they were 12 (50\%) males and 12 (50\%) females with an age range of 8 - 17 days. Additional 22 neonates, 12 (54.5\%) males and $10(45.5 \%)$ females, were recruited as high risk group, with ages ranging from 8 to 18 days, while the control group included 17 neonates $9(52.9 \%)$ males and 8 (47.1\%) females with an age range between 7 and 17 days. Other clinical and laboratory data of the studied groups are shown in Table (1).

Regarding risk factors and outcome, ten (41.7\%) of the proved sepsis group were on mechanical ventilation (MV), $4(16.7 \%)$ required central line and umbilical catheter (CL) insertion and 20 $(83.3 \%)$ required peripheral intravenous line insertion (IV). The risk factors for the high-risk group were maternal UTI either suspected $19(86.4 \%)$ or proved $2(9.1 \%)$ and PROM $1(4.5 \%)$. The outcome of the neonates was significantly different among the studied groups. While all $22(100 \%)$ high risk neonates survived, only 14 (58.3\%) of neonatal sepsis group survived and $10(41.7 \%)$ died.

In proved sepsis group, 4 (16.7\%) blood cultures showed no growth, 6 (gave gram negative bacilli (2 $(8.3 \%)$ were klebsiella and $4(16.6 \%)$ were E coli) and 14 showed gram positive cocci (12 (50\%) were Coagulase-Negative Staphylococci (CoNS) and $2(8.3 \%)$ were staph aureus).

As regards miR-146a expression, it showed significant differences between the studied groups. Figure (1).

Furthermore, analysis of correlations between miR-146a expression and the studied clinical /laboratory parameters, revealed that CRP had a significant positive correlation with miR-146a in high risk as well as proved sepsis groups $(\mathrm{p}<0.05$, both). Otherwise, no significant correlations were found ( $p>0.05$ all).

ROC curve analysis of data was performed to detect utility of miR-146a expression in the discrimination between high risk and proved sepsis groups. The best ROC curve was observed at cut off value of 0.539 relative units, with a sensitivity of $91.7 \%$, specificity of $100 \%$ and $95.7 \%$ accuracy. Table (2) and Figure (2) 
Table (1) Comparison between baseline clinical and laboratory features of all studied groups.

\begin{tabular}{|c|c|c|c|c|}
\hline & & $\begin{array}{c}\text { Control group } \\
(\mathrm{n}=17)\end{array}$ & $\begin{array}{l}\text { High risk group } \\
\quad(\mathrm{n}=22)\end{array}$ & $\begin{array}{l}\text { Proved sepsis group } \\
\qquad(\mathrm{n}=24)\end{array}$ \\
\hline & NVD & $7(41.2 \%)$ & $13(59.1 \%)$ & $8(33.3 \%)$ \\
\hline $\mathrm{n}(\%)$ & CS & $10(58.8 \%)$ & $9(40.9 \%)$ & $16(66.7 \%)$ \\
\hline & & & Median and $\mathrm{R}$ & \\
\hline Gestatio & (weeks) & $\begin{array}{c}38 \\
33-40 \\
\end{array}$ & $\begin{array}{c}34 \dagger \\
30-36\end{array}$ & $\begin{array}{l}30 \dagger+ \\
28-36\end{array}$ \\
\hline Weight & & $\begin{array}{c}2.75 \\
1.9-4.0\end{array}$ & $\begin{array}{c}2.0 \dagger \\
1.2-2.9\end{array}$ & $\begin{array}{l}2.7 \ddagger \\
1.7-3.5\end{array}$ \\
\hline $\begin{array}{l}\text { Total } \\
\left(\mathrm{X} 10^{9} / \mathrm{L}\right)\end{array}$ & tic count & $\begin{array}{c}8.4 \\
4-20.4 \\
\end{array}$ & $\begin{array}{c}8.4 \\
6-20.4 \\
\end{array}$ & $\begin{array}{c}10.4 \\
5.4-15\end{array}$ \\
\hline $\mathrm{Hb}$ conc & $(\mathrm{g} / \mathrm{dL})$ & $\begin{array}{c}15 \\
13.5-18.2\end{array}$ & $\begin{array}{c}12.5 \dagger \\
10.5-14.9\end{array}$ & $\begin{array}{c}11.55 \dagger \\
10.4-14.7\end{array}$ \\
\hline Platelet & $\left(10^{9} / \mathrm{L}\right)$ & $\begin{array}{c}264 \\
149-450\end{array}$ & $\begin{array}{c}100 \dagger \\
48-340\end{array}$ & $\begin{array}{c}97 \dagger \\
34-333 \\
\end{array}$ \\
\hline CRP tite & & ----- & $\begin{array}{c}12 \\
6-24\end{array}$ & $\begin{array}{c}48 \div \\
24-96\end{array}$ \\
\hline
\end{tabular}

n: total number, NVD: normal vaginal delivery, CS: Caesarian Section, Hb: Hemoglobin, $\uparrow:$ significant as compared to controls, $¥$ : significant as compared to high risk group, $\mathbf{P}<\mathbf{0 . 0 5}$ : significant

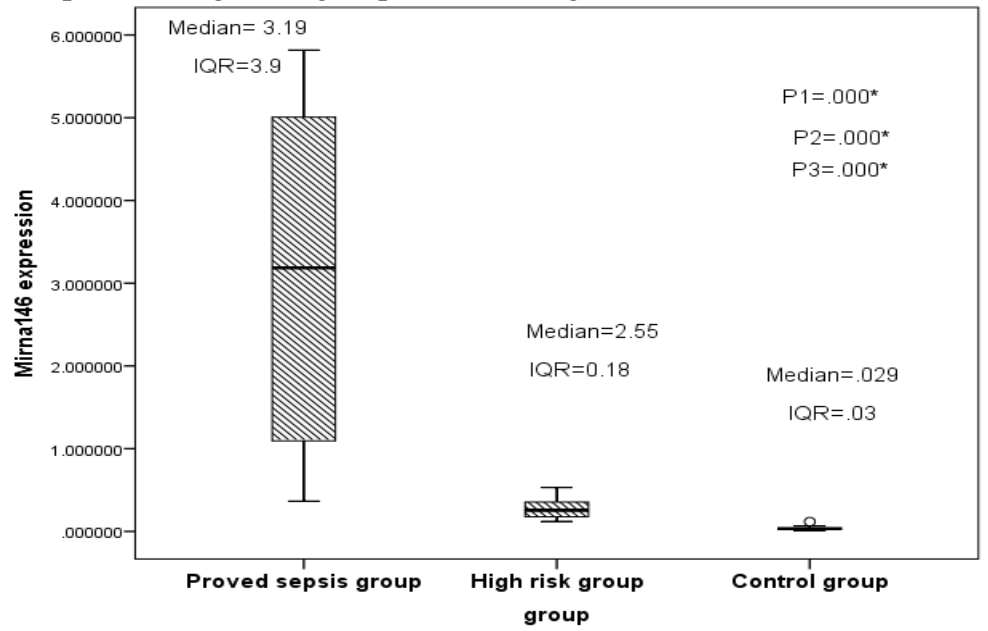

Figure (1). miR-146a expression among the studied groups. IQR: interquartile range, p1: proved sepsis \& high risk, p2: high risk \& controls, p3: proved sepsis \& Control, *: p<0.05 significant

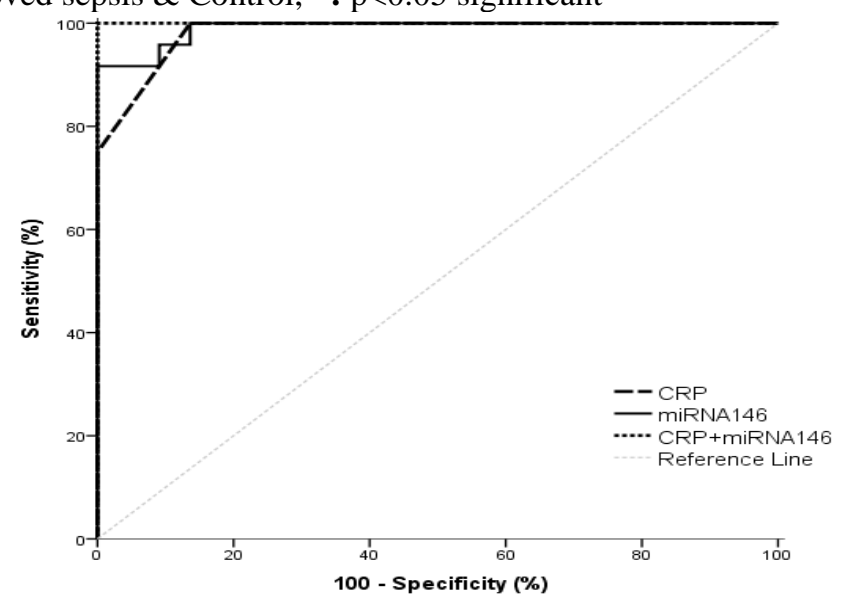

Figure (2). ROC curve of miR-146a expression for discrimination between high risk and proved sepsis groups. 
Table (2). AUC and performance characteristics of miR-146a expression, CRP and combination of both parameters in high risk and proved sepsis groups

\begin{tabular}{|c|c|c|c|}
\hline & miR-146a & CRP & miR146a + CRP \\
\hline Cut off & 0.539 & 18 & --- \\
\hline Sensitivity (\%) & 91.7 & 100 & 100 \\
\hline Specificity (\%) & 100 & 86.4 & 100 \\
\hline PPV (\%) & 100 & 88.9 & 100 \\
\hline NPV (\%) & 91.7 & 100 & 100 \\
\hline Accuracy (\%) & 95.7 & 93.5 & 100 \\
\hline AUC & 0.99 & 0.98 & 1 \\
\hline $95 \%$ CI & $0.97-1$ & $0.96-1$ & $1-1$ \\
\hline$P 1$ & $<0.001$ & $<0.001$ & $<0.001$ \\
\hline
\end{tabular}

Table (3). Regression analysis for prediction of sepsis within high risk group

\begin{tabular}{|l|c|c|c|c|c|c|c|c|}
\hline & \multicolumn{4}{|c|}{ Univariate } & \multicolumn{3}{c|}{ Multivariate } \\
\hline & $\boldsymbol{p}$ & OR & \multicolumn{2}{c|}{$\mathbf{9 5 \%}$ CI } & $\boldsymbol{p}$ & OR & \multicolumn{2}{c|}{ 95\% CI } \\
\hline Age (days) & 0.654 & 0.254 & 0.126 & 1.651 & & & & \\
\hline Male & 0.758 & 0.833 & 0.261 & 2.657 & & & & \\
\hline $\begin{array}{l}\text { Gestational } \\
\text { age (weeks) }\end{array}$ & 0.004 & 4.160 & 1.572 & 11.009 & 0.343 & 1.075 & 0.926 & 1.248 \\
\hline CS & 0.083 & 0.346 & 0.104 & 1.151 & & & & \\
\hline Weight (g) & 0.008 & 1.431 & 1.023 & 3.002 & 0.171 & 0.546 & 0.164 & 1.587 \\
\hline CRP & 0.038 & 1.407 & 1.999 & 2.015 & 0.042 & 1.387 & 1.027 & 2.651 \\
\hline miR-146a & 0.037 & 2.068 & 1.179 & 2.165 & 0.041 & 2.031 & 1.925 & 4.149 \\
\hline
\end{tabular}

$\boldsymbol{p}<0.05$ significant, OR: odds ratio, CI: confidence interval. CRP: C-reactive protein

Regarding CRP, the best ROC curve was at cutoff value of $18 \mathrm{mg} / \mathrm{dL}$ with $100 \%$ sensitivity, $86.4 \%$ and accuracy was $93.5 \%$. Interestingly, combination of miR-146a with CRP increased sensitivity, specificity and accuracy of CRP and miR-146a to $100 \%$. Table (2) and Figure (2).

In addition, there were no significant differences in miRNA146 expression in newborn with sepsis as regard to gender, mode of delivery, risk factors, outcome and type of organism identified by blood culture ( $p>0.05$ all).

Finally, logistic regression analysis was used for prediction of sepsis within high risk group using age, sex, gestational age, delivery mode, weight, CRP and miR-146a as covariates. Gestational age, weight and miR-146a were associated with risk of 
sepsis in univariate analysis. However, in multivariate analysis, only CRP and miR-146a were considered as independent risk factors for prediction of sepsis within high risk neonates (Table 3).

\section{Discussion}

The present study revealed a statistically significant increase in miR-146a serum expression level among proved sepsis group and high risk group neonates, as compared with healthy controls. In addition, miR-146a expression in sera of neonates with proved sepsis was significantly higher than in sera of the high-risk group neonates. These findings support previously reported results regarding upregulation of plasma miR-146a and miR-223 levels in children sepsis as compared with SIRS and control groups (12).

In agreement with the present data, Puskarich and colleagues (13) demonstrated higher plasma miR146a expression in sepsis patients as compared to those with septic shock or healthy controls, though the difference did not reach statistical significance level. More recently, a set of circulating miRNAs including miR-146a has been reported to increase in sepsis patients as compared to control (no-SIRS) patients (14).

Interestingly, the current data are consistent with the fact that transcription of miR-146a is induced in response to the bacterial endotoxin lipopolysaccharide (LPS) in a dose dependent manner (8, 15-17). Moreover earlier studies have shown that miR-146a was upregulated in immune competent cells in response to different bacterial infections including Mycobacteria species, Salmonella enterica and Helicobacter Pylori (15).
The mechanisms underlying miR-146a upregulation in sepsis have been also highlighted previously. The process starts by activation of the cell surface toll like receptors (TLR2, 4and 5) upon exposure to bacterial components. This is followed by induction of intracellular signaling through nuclear factor- $\kappa \mathrm{B}(\mathrm{NF}-\kappa \mathrm{B})$-dependent pathway. Of interest, $\mathrm{NF}-\kappa \mathrm{B}$ is a transcription factor which binds to promoters of many immune responsive genes including miR-146a, causing their induction $(\mathbf{7}, \mathbf{1 5}$, 16, 18, 19)

The role of miR-146a in sepsis has been investigated, as well. It has been suggested that miR-146a is implicated in fine adjustment of the innate immune response via a negative feedback mechanism. Former reports suggested that miR146a can downregulate TLR4 by direct binding to its target sequence on TLR4- $\operatorname{mRNA}(\mathbf{8}, \mathbf{2 0}, \mathbf{2 1})$. However, this direct mechanism has been controversial and accumulating evidence supports the role of miR-146a in targeting effector molecules in the TLRs pathway rather than TLRs themselves( (17).

Inconsistent with the current results, miR-146a was found to be significantly downregulated in sepsis patients than SIRS and normal control groups (22, 23). Such differences may be due to variation in patients' characteristics regarding the age, stage of sepsis, presence of co-morbidity or lines of treatment used in both studies.

The present study also showed a significant positive correlation between miR-146 and CRP titer in neonatal sepsis group. Previous studies on sepsis patients or animal models for sepsis documented that, in most cases, the course of sepsis starts with a proinflammatory phase which may then be 
followed by immune-suppression phase. During the proinflammatory phase, the innate immunity is activated with sensitization of immune cells, leukocytosis, activation of complement system, increased production of proinflammatory cytokines, chemokines, and release of CRP by liver (5).

However, Caserta and coworkers (14) reported an inverse correlation between a set of plasma miRNAs including miR-146a and CRP level in patients with sepsis. They suggested that the studied miRNAs play anti- inflammatory role and suppress immune cells in sepsis. Such contradiction may be explained by the different stages of sepsis in both studies, as well as, variations in patients' characteristics.

The ROC curve analysis revealed that miR-146a best cut off value was 0.539 , this value could predict sepsis, with area under the curve (AUC) of 0.991 and a confidence interval of $0.972-1$. miR146a had a sensitivity of $91.7 \%$, specificity $100 \%$, positive predictive value $100 \%$ and negative predictive value $91.7 \%$.

In this regard, these results agree with those of a former study on pediatric sepsis which showed that AUC of miR-146a was 0.815 and concluded that miR-146a may be a potential marker for early diagnosis of sepsis(12). In another study conducted on sepsis versus SIRS patients, miR-146a predicted sepsis with AUC of $0.813,60 \%$ sensitivity and specificity of $87.5 \%$. They also suggested the use of miR-146a as a marker to differentiate sepsis from SIRS (23).

Furthermore, ROC curve analysis of CRP at cutoff value of $18 \mathrm{mg} / \mathrm{dL}$ showed $100 \%$ sensitivity, $86.4 \%$ specificity and accuracy of $93.5 \%$. Importantly, the current study showed that, combination of both parameters, miR-146a and CRP increased the sensitivity, specificity and accuracy to $100 \%$.

However, a recent meta-analysis revealed that CRP had limited sensitivity (0.75) and specificity (0.67) to point out sepsis (8). Therefore, currently CRP is not recommended in guidelines as a biomarker for sepsis $(\mathbf{8}, \mathbf{2 4})$. Such discrepancy of results may be attributed to the difference in sample size.

Furthermore, the current study showed that only miR-146a and CRP were independent risk factors for prediction of sepsis within high risk neonates. This results suggest that miRNA may be promising for early identification of sepsis cases. To our knowledge, the results of logistic regression analysis regarding miR-146a expression in serum were not clearly mentioned in previous studies $\mathbf{( 8 ,}$ $12,23,25,26$ ).

Taking in consideration the limitation of the relatively small sample size, the results are promising and serum miR-146a may be a valuable predictor for sepsis within the high risk neonates, which may allow better management and safer outcome. Large-scale prospective studies are required to confirm our findings, with special emphasis on kinetics of miR-146a expression level during NS course and its relation to treatment.

\section{References}

(1) Fan Y and Yu JL: Umbilical blood biomarkers for predicting early-onset neonatal sepsis. World journal of pediatrics : WJP 8, 101-108, 2012.

(2) Simonsen KA, Anderson-Berry AL, Delair SF and Davies HD: Early-Onset 
Neonatal Sepsis. Clinical Microbiology

Reviews 27, 21-47, 2014.

(3) Shehab El-Din EMR, El-Sokkary MMA, Bassiouny MR and Hassan R: Epidemiology of Neonatal Sepsis and Implicated Pathogens: A Study from Egypt. BioMed Research International 2015, 509484, 2015.

(4) INAL Ç, TANRIÖVER MD and ERDEN DD: Novel Transcriptional Biomarkers for Diagosis and Prognosis of Sepsis. Virulence 5, 11-18, 2016.

Reinhart K, Bauer M, Riedemann NC and Hartog CS: New Approaches to Sepsis: Molecular Diagnostics and Biomarkers. Clinical Microbiology Reviews 25, 609-634, 2012.

(6) Choo YK, Cho H-S, Seo IB and Lee H-S: Comparison of the accuracy of neutrophil CD64 and C-reactive protein as a single test for the early detection of neonatal sepsis. Korean Journal of Pediatrics 55, 1117, 2012.

(7) Gulyaeva LF and Kushlinskiy NE: Regulatory mechanisms of microRNA expression. Journal of Translational Medicine 14, 143, 2016.

\footnotetext{
Benz F, Roy S, Trautwein C, Roderburg

$\mathbf{C}$ and Luedde $\mathbf{T}$ : Circulating MicroRNAs as Biomarkers for Sepsis. International Journal of Molecular Sciences 17, 78, 2016.
}

Li L, Chen XP and Li YJ: MicroRNA146a and Human Disease. Scandinavian Journal of Immunology 71, 227-231, 2010.

Dixon J, Bird H, Sitton NG, Pickup M and Wright V: C-reactive protein in the serial assessment of disease activity in rheumatoid arthritis. Scand J Rheumatol 13, 39-44, 1984.

Livak KJ and Schmittgen TD: Analysis of relative gene expression data using realtime quantitative PCR and the 2(-Delta Delta C(T)) Method. Methods (San Diego, Calif.) 25, 402-8, 2001.

Wu Y, Li C, He Y, Li Q, Wang G, Wen P, Yang W and Yang Y : Relationship between expression of microRNA and inflammatory cytokines plasma level in pediatric patients with sepsis. Zhonghua er ke $\mathrm{za}$ zhi $=$ Chinese journal of pediatrics 52, 28-33, 2014.

(13) Puskarich MA, Nandi U, Shapiro NI, Trzeciak S, Kline JA and Jones AE: Detection of microRNAs in patients with sepsis. Journal of Acute Disease 4, 101106, 2015.

(14) Caserta S, Kern F, Cohen J, Drage S, Newbury SF and Llewelyn MJ: Circulating Plasma microRNAs can differentiate Human Sepsis and Systemic Inflammatory Response Syndrome (SIRS). Scientific reports 6, 28006, 2016.

(15) Nahid MA, Pauley KM, Satoh M and Chan EK: miR-146a is critical for endotoxininduced tolerance: IMPLICATION IN 
INNATE IMMUNITY. The Journal of biological chemistry 284, 34590-9, 2009.

(16) Staedel C and Darfeuille F: MicroRNAs and bacterial infection. Cellular microbiology 15, 1496-507, 2013.

(17) Saba R, Sorensen DL and Booth SA: MicroRNA-146a: A Dominant, Negative Regulator of the Innate Immune Response. Frontiers in Immunology 5, 2014.

(18) Taganov KD, Boldin MP, Chang KJ and Baltimore D: NF-kappaB-dependent induction of microRNA miR-146, an inhibitor targeted to signaling proteins of innate immune responses. Proceedings of the National Academy of Sciences of the United States of America 103, 12481-6, 2006.

(19) Lorne E, Dupont $\mathbf{H}$ and Abraham E: Toll-like receptors 2 and 4: initiators of non-septic inflammation in critical care medicine? Intensive care medicine 36 , 1826-35, 2010.

(20) He Y, Sun X, Huang C, Long XR, Lin X, Zhang L, Lv XW and Li J: MiR-146a regulates IL-6 production in lipopolysaccharide-induced RAW264.7 macrophage cells by inhibiting Notch1. Inflammation 37, 71-82, 2014.

(21) Yang K, He YS, Wang XQ, Lu L, Chen QJ, Liu J, Sun $Z$ and Shen WF: MiR146a inhibits oxidized low-density lipoprotein-induced lipid accumulation and inflammatory response via targeting tolllike receptor 4. FEBS letters 585, 854-60, 2011.
-(22) Wang JF, Yu ML, Yu G, Bian JJ, Deng XM, Wan XJ and Zhu KM: Serum miR$146 \mathrm{a}$ and miR-223 as potential new biomarkers for sepsis. Biochemical and biophysical research communications 394, 184-8, 2010.

(23) Wang L, Wang H-C, Chen CHA, Zeng J, Wang Q, Zheng LEI and Yu H-D: Differential expression of plasma miR146a in sepsis patients compared with nonsepsis-SIRS patients. Experimental and Therapeutic Medicine 5, 1101-1104, 2013.

(24) Dellinger RP, Levy MM, Rhodes A, Annane D, Gerlach H, Opal SM, Sevransky JE, Sprung CL, Douglas IS, Jaeschke R, Osborn TM, Nunnally ME, Townsend SR, Reinhart K, Kleinpell RM, Angus DC, Deutschman CS, Machado FR, Rubenfeld GD, Webb SA, Beale RJ, Vincent JL and Moreno R: Surviving sepsis campaign: international guidelines for management of severe sepsis and septic shock: 2012. Critical care medicine 41, 580-637, 2013.

(25) Bloos F and Reinhart K: Rapid diagnosis of sepsis. Virulence 5, 154-160, 2014.

Dumache R, Rogobete AF, Bedreag OH, Sarandan M, Cradigati AC, Papurica M, Dumbuleu CM, Nartita $R$ and Sandesc D: Use of miRNAs as Biomarkers in Sepsis. Analytical Cellular Pathology 2015, $9,2015$. 